\title{
ESTIMULAÇÃO CEREBRAL NÃO-INVASIVA E EXERCÍCIOS FÍSICOS IMPACTAM VARIÁVEIS COGNITIVAS DE IDOSOS COM COMPROMETIMENTO COGNITIVO LEVE
}

\author{
Elisa Valente Barcelos ${ }^{(1 ; 2)}$; Deborah Casarsa ${ }^{(3)}$; Gilson Oliveira Filho ${ }^{(4)}$ Daniel Delani $^{(5)}$ \& \\ Mauricio Rocha Calomeni ${ }^{\left(1^{*}\right)}$
}

\begin{abstract}
RESUMO
BARCELOS, E.V. et al. Estimulação cerebral não-invasiva e exercícios físicos impactam variáveis cognitivas de idosos com comprometimento cognitivo leve. Perspectivas Online: Biológicas \& Saúde, v.11, n.37, p. 36-49, 2021.
\end{abstract}

O estudo verificou o efeito da estimulação cerebral associada aos exercícios com alta demanda cognitiva em variáveis cognitivas e atividade cerebral de idosos com CCL. 18 idosos de ambos os gêneros, idade igual ou superior a 60 anos, diagnosticados com CCL foram divididos em Grupo Controle (GC, n=8); Grupo Experimental (GE, $\mathrm{n}=10$ ). Foram avaliados o tempo de reação, memória de trabalho e potência relativa das ondas cerebrais. O GC permaneceu frequentando reuniões de treinamento da memória. O GE teve estimuladas as ondas cerebrais Alfa associado a atividades motoras que exigiam alta demanda cognitiva. Foram feitas sessões semanais de 15 min de estimulação cerebral +25 min de atividades motoras durante 7 semanas. Após esse período os indivíduos tiveram novamente as variáveis avaliadas. Teste $\mathrm{T}$ foi utilizado nas comparações e verificou-se de forma descritiva que o GE obteve um desempenho melhor que o grupo controle nos testes. Na potência das ondas cerebrais Teta e Delta o GC registrou aumento Teta nas áreas parietais, e Delta tanto nas áreas parietais quanto frontais. O GE teve diminuição da potência da onda Teta nas áreas parietais e frontais. A potência da onda Alfa diminuiu em ambos os grupos, com menor redução no GE. Nenhuma das comparações entre os grupos se mostrou significantes estatisticamente. Conclui-se que a associação dos estímulos físicos e cognitivos aplicados em sessões semanais de 40 minutos durante 7 semanas não foi suficiente para produzir resultados estatisticamente significativos. Contudo, os resultados descritivos observados se assemelham aos de outros que apontam a eficiência de treinamentos que associem estímulos físicos e cognitivos durante períodos de intervenção mais prolongados.

Palavras-chave: Comprometimento cognitivo leve; Demência; Exercício físico; Estimulação cerebral.

\footnotetext{
(1) Laboratório de Biociências da Motricidade Humana -LABIMH/ISECENSA; (2) Bolsista PIBIC/CNPQ/ISECENSA - Curso de Educação Física; ${ }^{(3)}$ Médica Geriatra, especialista em Gerontologia e Geriatria; ${ }^{(4)}$ Universidade do Estado do Rio De JaneiroUERJ; ${ }^{(5)}$ Departamento de Educação Física da Universidade Federal de Rondônia

(*) e-mail: mauriciocalomeni@gmail.com

Data de recebimento: 28/0/2021. Aceito para publicação: 21/06/2021.

Data de publicação: 30/06/2021.
} 


\section{NON-INVASIVE BRAIN STIMULATION AND PHYSICAL EXERCISE IMPACT COGNITIVE VARIABLES OF ELDERLY WITH MILD COGNITIVE IMPAIRMENT Elisa Valente Barcelos ${ }^{(1 ; 2)}$; Deborah Casarsa ${ }^{(3)}$; Gilson Oliveira Filho ${ }^{(4)}$; Daniel Delani $^{(5)}$ \& Mauricio Rocha Calomeni ${ }^{\left(1^{*}\right)}$}

\section{ABSTRACT}

BARCELOS, E.V. et al. Estimulação cerebral não-invasiva e exercícios físicos impactam variáveis cognitivas de idosos com comprometimento cognitivo leve. Perspectivas Online: Biológicas \& Saúde, v.11, n.37, p. 36-49, 2021.

The study verified the effect of brain stimulation associated with physical exercises with high cognitive demand in cognitive variables and brain activity of aged with MCI. 18 elderly of both genders, age equal or upper to 60 years, diagnosed with MCI were divided between Control Group ( $\mathrm{CG}, \mathrm{n}=8$ ); Experimental Group $(\mathrm{EG}, \mathrm{n}=10)$. Were evaluated the reaction time, working memory, and relative potency of brainwaves. The CG kept their regular activities in the memory training group. The EG got stimulated the Alpha brainwaves conjugated with the motor tasks that demanded high cognitive activity. Were made once week 15 minutes of brain stimulation plus 25 minutes of motor tasks per 7 weeks. After this time the participants got the variables evaluated again. T-test was used in comparisons and was verified in a descriptive way that the EG got better performance than $\mathrm{CG}$ in the tests. In the relative potency of Theta and Delta brainwaves, the CG got Theta-increase in parietals areas and Delta-increase both parietals and frontal areas. The EG got a decrease in Theta relative potency both in the parietals and frontal areas. Alphapotency decrease in both groups, with a lower reduction in the EG. No comparisons between groups were statistically significant. We conclude that the association of physical and cognitive stimulus once a week by 40 minutes in 7 weeks wasn't enough to produce statistically significant results. However, the observed descriptive data agree with other researches that shown the effectiveness of the association of physical and cognitive stimulus for a longer time.

Keywords: Mild Cognitive Impairment; Dementia; Physical Exercises; Brain Stimulation.

\footnotetext{
(1) Human Motricity Bioscience Laboratory -HMBL/ISECENSA; (2) PIBIC/CNPQ/ISECENSA Scholarship - Physical Education Course; (3) Geriatrician, specialist in Gerontology and Geriatrics; ${ }^{(4)}$ Rio De Janeiro State University - UERJ; Department of Physical Education, Rondonia Federal University. (*) e-mail: mauriciocalomeni@gmail.com Received: 28/0/2021. 


\section{INTRODUÇÃOO}

O envelhecimento é um processo que acontece de forma natural e espontânea com todos os seres humanos, por esse motivo é um assunto que tem ganhado cada vez mais a atenção de muitos pesquisadores e estudiosos ao redor do mundo, pois os níveis de desenvolvimento de um país são parcialmente determinados pela composição de sua população, uma vez que grupos etários diferentes têm impactos específicos no ambiente e nas necessidades de infraestrutura (CAMARANO et al, 2013).

Para Souza et al (2020) o envelhecimento populacional é um fenômeno global. Concomitantemente a este fenômeno percebe-se também um aumento dos casos de demência (MINAYO, et al, 2019), que se caracterizam por uma série de eventos que culminam na degeneração de neurônios relacionados a funções cognitivas e motoras (TEIXEIRA et al 2021). Todavia, a passagem de um estado de envelhecimento que seja sadio para um processo de perdas das funções cognitivas é mediado pela instalação de um estado de Comprometimento Cognitivo Leve (CCL), que pode ou não evoluir para quadros de demência (JERÔNIMO, 2018). Sendo assim, agravamentos do CCL que são caracterizados pela dificuldade para encontrar palavras, desorientação no tempo e no espaço e dificuldades para tomar decisões são fortes indícios de instalação de estados de demência (LIMA et al, 2014).

Então, o grande desafio que surge desse cenário é encontrar formas de se evitar que os sintomas do CCL evoluam para o estado incurável da demência. Nesse sentido, formas não farmacológicas como as estimulações motora (exercícios físicos) e cognitiva têm sido testadas cientificamente tanto de forma isolada quanto associada, para se promover benefícios em indivíduos com comprometimento cognitivo (LIMA NETA et al, 2020).

Tanto a estimulação motora (COELHO et al, 2009; MAIA et al, 2019) quanto a estimulação cognitiva (FERNANDES, 2014; LOUSA 2016) utilizados isoladamente têm se sido eficaz na prevenção e tratamento do CCL e da Demência. Contudo, uma série de estudos tem verificado que a associação das estimulações motora e cognitiva tem mostrado resultados ainda mais promissores (STYLIADIS et al 2015; CONSORTIUM, 2017; OKAMURA et all, 2018). Além disso, evidências apontam uma relação entre frequências de ondas cerebrais como Teta, Delta e Alfa com a evolução do CCL (MARLATS et al 2019; RADIĆ et all, 2019), bem como o efeito de arrastamento que a estimulação cerebral não invasiva por batidas binaurais pode ter sobre essas ondas (CALOMENI et al, 2017; SILVA VERNON et al, 2019).

Sendo assim, torna-se importante que se verifique científicamente se a conjugação de protocolos de estimulação física e cognitiva associados com a estimulação cerebral não invasiva podem produzir efeitos positivos semelhantes aos observados nos estudos que verificaram essas técnicas isoladamente. Assim, o presente estudo tem como objetivo verificar como um protocolo de estimulação física e cognitiva associado à estimulação cerebral não invasiva por batidas binaurais pode impactar o desempenho em testes cognitivos e a potência de ondas cerebrais de idosos com declínio cognitivo leve (CCL).

Persp. Online: biol. \& saúde, Campos dos Goytacazes, 37 (11) 36-49, 2021 


\section{METODOLOGIA}

O estudo trata-se de um ensaio clínico controlado. Este tipo de estudo verifica o efeito de determinada intervenção em um grupo experimental, geralmente terapêutica, quando comparada a controles (ESCOSTEGUY, 2014). O projeto foi avaliado e aprovado pelo Comitê de Ética do ISECENSA, com parecer consubstanciado $\mathrm{n}^{\circ}$ 3.464.436, e registrado na plataforma brasileira de registro de Ensaios Clínicos (ReBEC) com nº UTN: U1111-1249-0494.

Para compor a amostra deste estudo foram selecionados por conveniência 20 voluntários de um universo de 56 idosos atendidos pelo centro de doença de Alzheimer e Parkinson (CDAP), mantido pelo poder municipal de Campos dos Goytacazes/RJ, com diagnóstico de declínio cognitivo leve registrado em seu prontuário. Os voluntários foram divididos em dois grupos, 10 no grupo experimental (GE) e 10 no grupo controle (GC). Em ambos os grupos foram incluídos idosos que participavam regularmente de uma oficina de estimulação cognitiva oferecida no CDAP, com idade igual ou superior a 60 anos, de ambos os sexos e que concordaram voluntariamente em participar da pesquisa.

Foram excluídos idosos que por quaisquer limitações físicas estivessem incapacitados de executar as tarefas nos protocolos da pesquisa, e aqueles que apresentassem frequência inferior a $60 \%$ nas sessões de intervenção. Sendo assim, durante o período de intervenção do estudo 2 idosos do grupo controle foram excluídos por não manterem frequência superior a $60 \%$ na oficina de treinamento da memória. Dessa forma, a amostra final do estudo foi de 18 participantes, sendo 10 no grupo experimental $(n=10)$ e 8 no grupo controle $(n=8)$.

A funcionalidade da memória de trabalho foi determinada pelo Digit Span que é um subteste da escala WAIS-III (NASCIMENTO, 2004), cuja validade já foi determinada para a avaliação do declínio cognitivo de idosos (PAULA et al, 2010). O Digit Span é um teste que que verifica a extenção da memória de trabalho através de aferição do seu aspecto funcional. $\mathrm{O}$ teste se inicia com o examinador solicitando ao paciente que repita uma série de números, sendo que, a primeira sequência começa com dois dígitos. Após cada resposta correta acrescenta-se um dígito na sequência seguinte. Foi solicitado que os participantes apenas repetissem as sequências de dígitos na ordem direta, sendo o teste interrompido quando o avaliado errava uma sequência de dígitos que foram geradas aleatoriamente por uma versão computadorizada do Digit Span desenvolvido por Jared Blackburn@ em 2011. Trata-se de um software de licença livre, disponível na internet, especificamente criado para informatizar a aplicação do Digit Span.

Para a mensuração da velocidade de processamento mental foi utilizado um teste gratuito, disponível na internet, desenvolvido por Okazaki (2011) e que possui a finalidade de registrar o tempo de reação, considerando o intervalo de tempo entre a apresentação de determinado estímulo e a resposta motora apropriada. É um teste de fácil aplicação, que depende somente de um notebook, podendo utilizar diferentes tipos de estímulos, como letras, números, símbolos ou cores. Segundo Gonçalves et al. (2014), a resposta motora envolve todas as etapas necessárias do processamento mental, logo, inferências sobre este processamento podem ser realizadas através do tempo de reação. Isso é possível pois a velocidade da resposta motora depende de aspectos específicos como percepção do estímulo, interpretação deste, programação da resposta e execução da resposta apropriada. Conforme já exposto na literatura (ROSSATO et al., 2011), a manutenção da capacidade física funcional quando se trata de idosos é uma resposta que pode ser alterada mediante a estimulação, a partir da verificação do tempo

Persp. Online: biol. \& saúde, Campos dos Goytacazes, 37 (11) 36-49, 2021

seer.perspectivasonline.com.br 
de reação em relação ao processamento mental.

A avaliação da atividade cerebral foi realizada por um aparelho de eletroencefalograma que registra a atividade elétrica em pontos específicos do córtex cerebral em tempo real. Para tal avaliação foi utilizado o equipamento produzido pela Neurotec, no Brasil, modelo Neuromap EQSA260, versão 3.1.0. Todos os pontos avaliados foram determinados em conformidade com o sistema internacional 10/20 indicado pela Sociedade Brasileira de Neurofisiologia Clínica (SILVA et al., 2016). Em relação à efetividade deste instrumento, todos os cuidados foram tomados conforme os procedimentos adotados por Branco et al. (2020) para um perfeito aterramento do instrumento e assepsia dos pontos de referência dos eletrodos no escalpo, de forma a manter a taxa de impedância dentro dos valores mínimos aceitáveis nas recomendações do aparelho e a evitar quaisquer interferências elétricas durante a coleta dos dados. Alguns equipamentos semelhantes têm sido utilizados em estudos variados na área da neurociência com o mesmo objetivo de mensurar a atividade elétrica no córtex cerebral (MARQUES et al., 2006; CALOMENI et al., 2013; CALOMENI et al., 2017).

Para produção das batidas binaurais, necessárias à estimulação cerebral não invasiva, foi utilizado um aparelho eletrônico sintetizador de ondas corticais, modelo Sirius, fabricado pela Mind Place. Foi utilizada uma sessão de treinamento específica para induzir estados de concentração com duração de 15 min. O áudio da referida sessão foi convertido em arquivo MP3 para que pudesse ser utilizada em qualquer aparelho de som que disponibilizasse essa função. Tal instrumento trata-se de um estimulador não-invasivo de ondas cerebrais que já foi utilizado em estudos que investigam sua mediação em termos de funções mentais (CARIELO et al., 2010; ARÊAS NETO et al., 2010; CALOMENI et al., 2013; SILVA VERNON et al., 2015; CALOMENI et al., 2017).

As avaliações iniciais foram feitas em salas cedidas pelo CDAP que foram devidamente preparadas para minimizar o efeito de detratores como ruído excessivo e temperatura elevada que poderiam comprometer a fidedignidade dos dados. Os participantes foram convidados individualmente a entrar nas referidas salas para que os testes pudessem ser aplicados de acordo com as especificações de cada protocolo.

Inicialmente, foi solicitado que os participantes se sentassem o mais confortavelmente possível para que os eletrodos para o registro da atividade cerebral fossem posicionados no escalpo. A localização de cada eletrodo se deu de acordo com o sistema internacional 10/20 (JURCAK; TSUZUKI; DAN; 2007), e logo após a localização de cada ponto foi feita a assepsia com algodão e solução de álcool a $70^{\circ}$. Com todos os eletrodos posicionados era verificado se a taxa de impedância estava abaixo de $20(\mathrm{~K} \Omega)$, para que se pudesse ser iniciada a coleta de dados que tinha duração total de 3 minutos para se determinar uma linha de base da atividade cerebral de cada participante. Os pontos de interesse do estudo foram os pontos F7 que está relacionado com a memória de trabalho visual e auditiva, atenção seletiva e dividida, F8 relacionado à memória de trabalho visual e espacial, processamento emocional e manutenção da atenção, e por fim os pontos P3 e P4 relacionados à resolução de problemas, atenção e associação, processamento visual e associação não verbal (SOUTAR e LONGO, 2011). Além destes foram utilizados os pontos A1 e A2 como referência e mais 1 ponto como terra.

Após determinação do padrão de atividade cerebral as avaliações seguintes foram a de velocidade de processamento mental através do tempo de reação motora e da memória de trabalho através do Digit Span, respectivamente. Para essas avaliações, o participante foi

Persp. Online: biol. \& saúde, Campos dos Goytacazes, 37 (11) 36-49, 2021

seer.perspectivasonline.com.br 
posicionado na frente de um computador onde estavam instalados os softwares específicos para tais avaliações. Como procedimento padrão, após as explicações da dinâmica das avaliações foi permitido que o participante fizesse algumas avaliações teste em cada um dos softwares, de forma que se familiarize com os procedimentos e fossem minimizadas a chance de erros durante a coleta dos dados. Todos os procedimentos até agora descritos foram destinados à determinação do status pré e pós intervenção das variáveis avaliadas no estudo.

A intervenção com os exercícios com alta demanda cognitiva aplicada nos participantes do grupo experimental consistiu de atividades baseadas no lúdico, que estimulam a coordenação motora, o equilíbrio e a força funcional dos idosos, com intensidade moderada a baixa de acordo com a percepção individual de esforço, de forma que não promovesse um desgaste excessivo nos participantes. Todavia, tais exercícios foram associados a outras atividades de ordem cognitiva como o armazenamento, retenção e recuperação de informações, e tomada de decisão de acordo com inputs externos, além da prática de mentalização dos exercícios propostos antes realização dos mesmos.

Associados aos exercícios com alta demanda cognitiva foi aplicado o protocolo de estimulação cerebral por batidas binaurais como procedimento auxiliar. As batidas binaurais eram produzidas por um aparelho eletrônico denominado Brain Machine fabricado pela Mind Place que possui sessões pré-programadas de estimulação cerebral destinadas a promover estados de alerta e concentração. Ou seja, a prática dos exercícios físicos com alta demanda cognitiva foi conjugada a um protocolo de estimulação cerebral não invasiva por batidas binaurais, que promovia o estímulo e treinamento de ondas cerebrais específicas.

O período de intervenção no grupo experimental teve um total de 7 semanas, sendo as sessões de treinamento feitas 1 vez por semana com duração total de 40 minutos, sendo que os primeiros 15 minutos foram destinados ao protocolo de estimulação cerebral não invasiva, e os 25 minutos restantes à prática dos exercícios com alta demanda cognitiva. Durante todo o período de intervenção no grupo experimental os idosos do grupo controle permaneceram com suas atividades normais, que incluíam a participação nas atividades da oficina de memória que já era oferecida no CDAP. Após o período de intervenção, todas as avaliações foram realizadas novamente, seguindo o mesmo padrão.

A metodologia de análise de dados foi feita preliminarmente por meio da normalização dos resultados, que permitiu determinar valores percentuais de performance de cada indivíduo da avaliação pós-intervenção para a pré-intervenção. Determinados o percentual de desempenho em cada variável, foram determinadas a tendência central, os extremos e o desvio padrão dos escores. A análise inferencial foi feita através da aplicação de testes T não-pareados para a análise intergrupos. Foi adotado nível de confiança de $95 \%$ para aceitação dos resultados $(\mathrm{p}<0,05)$.

\section{RESULTADOS E DISCUSSÃO}

Para tentar atender ao objetivo do estudo os primeiros dados que serão apresentados são relativos ao desempenho dos participantes nos testes cognitivos previstos na metodologia. Isso será feito por meio de um gráfico que apresentará de forma visual tanto informações descritivas quanto os valores de $\mathrm{p}$ referentes à análise inferencial que apontará se as diferenças nas comparações são estatisticamente significativas.

Persp. Online: biol. \& saúde, Campos dos Goytacazes, 37 (11) 36-49, 2021

seer.perspectivasonline.com.br 
Testes Cognitivos

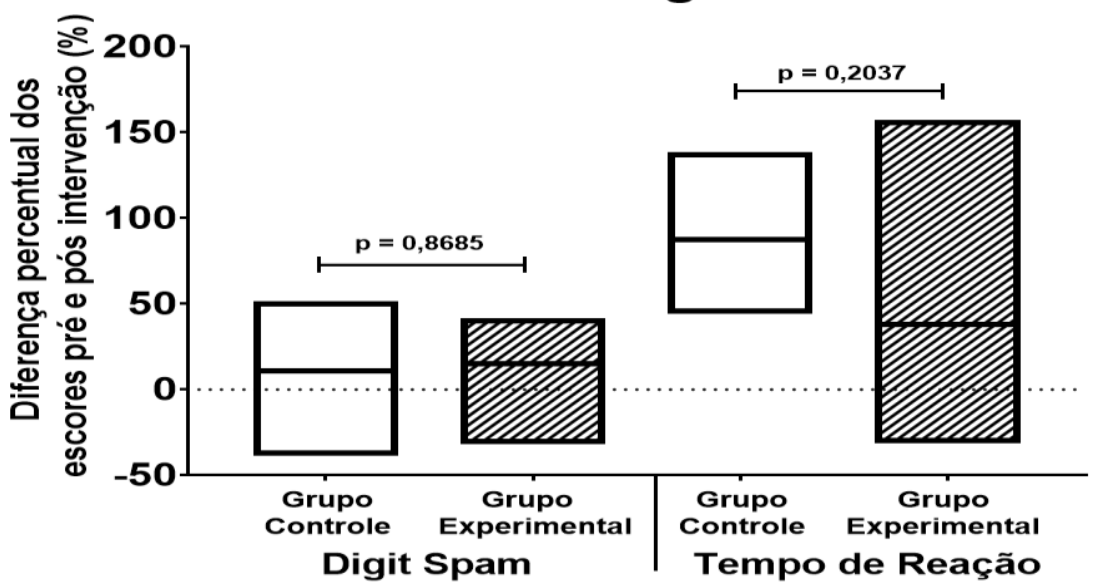

Figura 1. Apresentação do percentual de desempenho nos testes digit span e tempo de reação e dos participantes dos grupos controle e experimental. Escores negativos indicam desempenho inferior na avaliação pós-intervenção quando comparado à avaliação préintervenção. Em todas as comparações os valores de p indicam que as diferenças entre os grupos não foram estatisticamente significativas.

É possível verificar que apesar de não significativas estatisticamente registrou-se mudanças no desempenho dos participantes nos testes cognitivos que parecem ser interessantes. Analisando o comportamento dos grupos no teste Digit Span nota-se que ambos os grupos foram mais eficientes nas avaliações pós-intervenção, havendo uma diferença de 36,9\% entre os grupos. Em outras palavras ambos os grupos melhoraram o desempenho da memória de trabalho, fato compreensível uma vez que os participantes dos dois grupos tiveram essa função estimulada através de meios diferentes. Netto et al (2013) analisaram o efeito de um programa de treinamento da memória de trabalho em 11 idosos saudáveis, para isso também utilizaram o Digit Span como um dos instrumentos de avaliação e verificaram diferenças significativas no desempenho da memória de trabalho após 3 meses com 12 sessões semanais de treinamento com duração de 90 minutos. Já o estudo de Costa e Sequeira (2013) valeu-se como principal estratégia para o programa de estimulação cognitiva, o treinamento da memória de trabalho em 17 idosos com declínio cognitivo leve durante 8 semanas, sendo 1 sessão semanal também de 90 minutos. Assim como no estudo de Netto et al, o estudo de Costa e Sequeira também registrou melhora da memóra de curto prazo no grupo experimental, o que resultou em melhora significativa na utilização de estratégias de memória. Ao que parece no que diz respeito a incrementos significativos em memória o tempo da sessão de treinamento parece ter papel determinante, uma vez que os dois estudos supracitados tiveram tempos de intervenções distintos, porém com mesmo tempo de duração das sessões (90'), o que foi o dobro do tempo utilizado nas sessões de intervenção do presente estudo.

Também na figura 1 ficam evidenciadas variações no desempenho no teste de tempo de reação motora. Os dois grupos registraram aumento na velocidade de reação motora, o que significa um pior desempenho na velocidade de processamento de informação. Todavia, diferentemente do teste de memória de trabalho, a diferença entre os grupos ficou mais acentuada $(56,6 \%)$, sendo que o pior desempenho foi do grupo controle com aumento de $87,4 \%$,

Persp. Online: biol. \& saúde, Campos dos Goytacazes, 37 (11) 36-49, 2021

seer.perspectivasonline.com.br 
enquanto que no grupo experimental o aumento foi de 37,9\%. Tais resultados podem indicar eficiência da estimulação feita no grupo experimental sobre essa variável. Pois, por se tratar de idosos diagnosticados com declínio cognitivo era de se esperar declínio da velocidade de processamento de informação, e consequentemente do tempo de reação motora. Rossato, Contreira e Corazza (2011) respaldando-se em estudos como os de Santos e Tani (1995) afirmaram que as perdas e declínios associados ao envelhecimento aumentam o tempo de resposta motora. Além disso, Gonçalves et al (2014) utilizaram instrumento semelhante ao do presente estudo para avaliar o tempo de reação motora de 10 idosos insititucionalizados e encontraram uma correlação forte $(-0,72)$ entre maiores tempos de reação motora e níveis mais baixos de atividade cognitiva. Dessa forma, fica evidente que após o período de intervenção os participantes do grupo controle estavam com a capacidade de processamento de informações, e por conseguência a capacidade cognitiva, mais comprometida que os participantes do grupo experimental, pois apresentaram maiores tempos de resposta motora.

Complementando as avaliações propostas, foi determinada a potência relativa das ondas cerebrais Teta, Delta e Alfa. Antes de discutir efetivamente os dados encontrados é importante ressaltar que essas ondas cerebrais selecionadas são diretamente, e diferentemente, afetadas em indivíduos com declínio cognitivo e demência, pois em estados de CCL progressivo e doença de Alzheimer provável ocorre diminuição da atividade Alfa e aumento da atividade de ondas lentas (Teta e Delta) (LUCKHAUS et al., 2008). Sendo assim, na figura 2 será possível observar o comportamento dessas ondas cerebrais nos participantes dos grupos da pesquisa em termos percentuais.
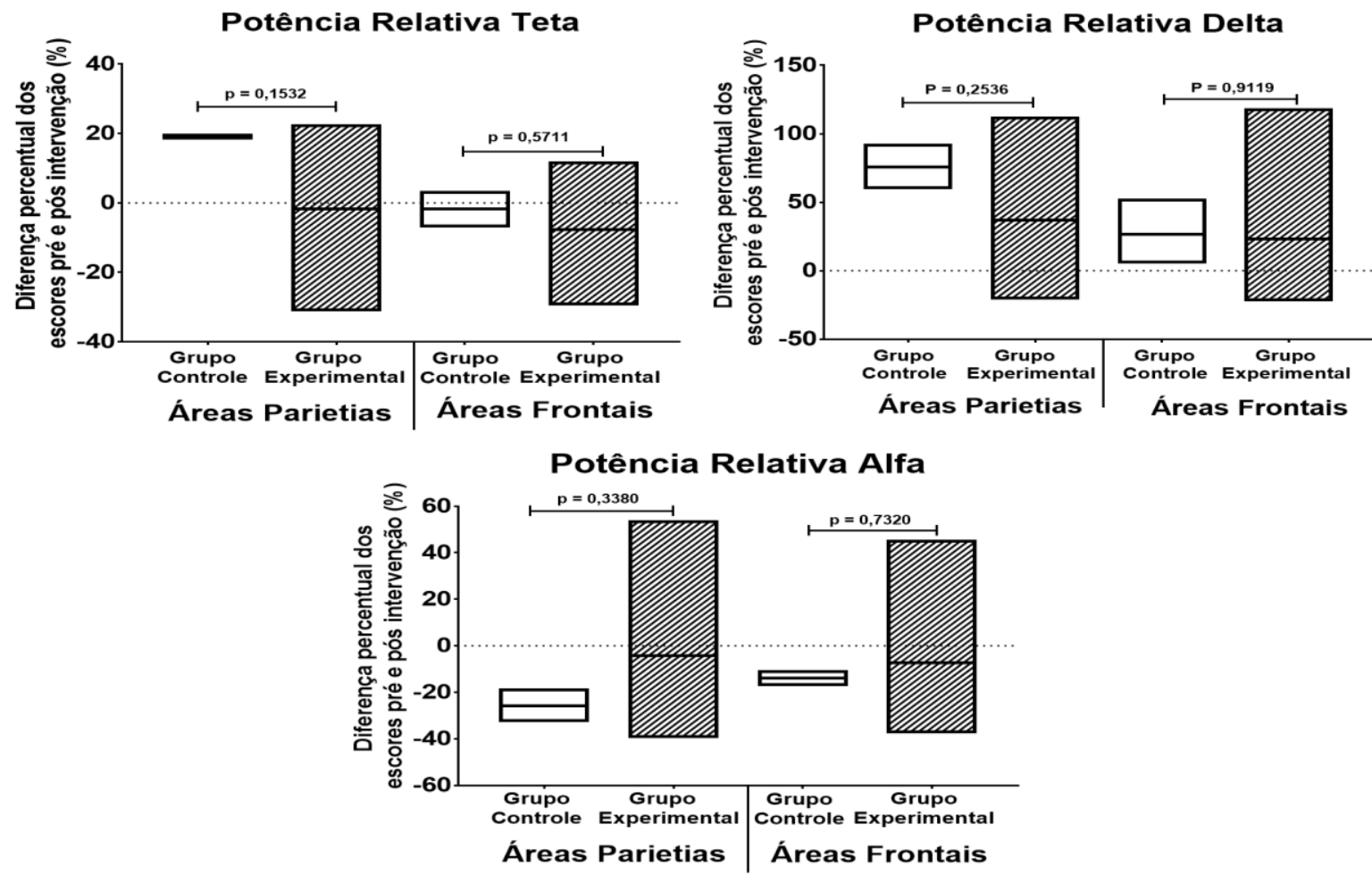

Figura 2. Apresentação do comportamento das ondas cerebrais Alfa, Teta e Delta nos grupos controle e experimental. Os dados representam a variação percentual entre os escores obtidos nas avaliações pré e pós-intervenção. Os escores negativos indicam que a potência relativa medida após a intervenção foi menor que a mesma medida da potência feita antes da

Persp. Online: biol. \& saúde, Campos dos Goytacazes, 37 (11) 36-49, 2021 seer.perspectivasonline.com.br 
intervenção. Os valores de $\mathrm{p}$ indicam que em nenhuma comparação as diferenças registradas foram estatisticamente significativas.

Apesar de também não significantes estatisticamente, os dados descritivos plotados nos gráficos da figura 2 corroboram com os achados apresentados na literatura com relação potência das ondas analisadas e estados de declínio cognitivo e demência, ou seja, com relação às ondas cerebrais mais lentas (Teta e Delta) os participantes do grupo controle registraram aumento da potência de Teta nas áreas parietais $(19,1 \%)$ e na potência da onda Delta tanto nas áreas parietais $(75,6 \%)$ quanto nas áreas frontais $(26,6 \%)$. A única área em que se registrou pequena redução da potência relativa da onda Teta foi na área frontal dos participantes do grupo controle $(-1,7 \%)$. Por outro lado, o grupo experimental teve diminuição da potência relativa da onda Teta no registro pós-intervenção nas áreas parietais $(-1,8 \%)$ e também nas áreas frontais $(-7,6 \%)$, além disso, os aumentos registrados na potência da onda Delta foram menores dos que os registrados na potência dessa mesma onda no grupo experimental, 36,8\% e 22,9\% nas áreas parietais e frontais respectivamente.

Por fim, a figura 2 traz também a plotagem dos dados da potência relativa da onda Alfa. Nessa nota-se que nos dois grupos a potência da onda Alfa após as intervenções diminuiu como era de se esperar devido à presença do CCL em todos os participantes. Todavia, a redução da potência relativa registrada no grupo controle nas áreas parietais $(-25,8 \%)$ e frontais $(-14 \%)$ foram maiores que as registradas no grupo experimental nas áreas parietais $(-4,2 \%)$ e frontais $(-7,3 \%)$. Apesar dos dados referentes à potência da onda Alfa não serem satisfatórios em ambos os grupos, mais uma vez é possível de se dizer que os resultados do grupo experimental foram melhores, pois mostram um declínio menor da potência dessa onda cerebral. Em um estudo que envolveu em sua amostra 15 idosos diagnosticados com Alzheimer, Calomeni et al (2017) verificaram o efeito agudo da estimulação por batidas binaurais associada a estimulação por luz stroboscópica no arrastamento das ondas cerebrais Alfa e SMR. Esses autores verificaram um aumento de $97 \%(\mathrm{p}=0,0005)$ na atividade elétrica em milivolts da onda Alfa no ponto F7 após 10 sessões de 15 minutos de estimulação cerebral não invasiva associado a exercícios de memória. Diferentemente deste estudo, a pesquisa desenvolvida por Calomeni e seus colaboradores utilizaram fones de ouvidos e óculos devidamente preparados para emitir tanto o som binaural quanto a luz strobo de forma mais concentrada nos participantes do estudo, o que pode justificar o efeito de neuromodulação observado.

Caranelli et al (2011) em um estudo sobre diagnóstico da doença de Alzheimer no Brasil se embasam em autores mais antigos para afirmar que no EEG os achados mais comuns são "(...) aumento das bandas delta e teta, e a diminuição ou abolição da banda de frequência alfa". Tal assertiva é corroborada por Pineda (2019) que amplia essa concepção afirmando que os estágios iniciais de DA são caracterizados por aumento da atividade Teta e diminuição da atividade das ondas Beta, e, com a progressão da doença ocorre também diminuição da atividade das ondas Alfa e, nos estágios mais avançados ocorre o aumento da atividade Delta. Diante destes fatos, mais uma vez, os dados do eletroencefalograma apontam para uma maior efetividade da intervenção feita no grupo experimental, haja vista que podem caracterizar uma evolução do declínio cognitivo menos acelerado quando confrontados com os mesmos dados do grupo controle.

Os resultados apresentados além de corroborar com o que é encontrado na literatura sobre a evolução do declínio cognitivo para demência deixam claro que, ao menos durante o

Persp. Online: biol. \& saúde, Campos dos Goytacazes, 37 (11) 36-49, 2021 seer.perspectivasonline.com.br 
período de intervenção do estudo, os participantes do grupo experimental possivelmente tiveram um efeito agudo de desaceleração desse processo. De fato são cada vez mais robustas as evidências de que a técnica de estimulação cerebral não invasiva utilizada aqui pode ser associada a outras intervenções para produzir efeitos satisfatórios na atenção concentrada de crianças diagnosticadas com TDAH (LIMA et al, 2014; CALOMENI et al, 2018), na redução da propabilidade de queda e no equilíbrio em idosos frágeis (SILVA VERNON et al, 2019; VERNON et al, 2020), assim como também a associação da estimulação física e cognitiva, aqui definida como exercícios físicos com alta demanda cognitiva, tem produzido rezuldados promissores verificados nos trabalhos de Styliadis et al (2015), Consortium et al (2017), Mcewen et al (2018), e, Okamura et al (2018). Dessa forma, parece plausível presumir que a junção da estimulação cerebral não invasiva com os exercícios com alta demanda cognitiva pudessem culminar em resultados estatisticamente significativos caso fosse possível mais algumas semanas de intervenção no grupo experimental.

Por fim, um ponto que se precisa levar em conta é que os resultados apresentados e discutidos foram obtidos após apenas 7 semanas de intervenção. Uma vez que, devido pandemia de COVID-19 não foi possível um tempo maior de intervenção. Outra limitação do estudo é que devido ao universo relativamente pequeno de idosos atendidos no CDAP, e a um número ainda menor de voluntários para o estudo, não foi possível a criação de um grupo de idosos para ser submetido apenas aos exercícios com alta demanada cognitiva o que permitiria verificar os efeitos desses exercícios associados ou não à estimulação cerebral não invasiva.

\section{CONCLUSÕES}

Todas as apresentações e considerações feitas a partir dos dados observados neste estudo não permitem a confirmação clara de uma hipótese para o estudo, pois, a falta de significância estatística nas comparações feitas apontam para a não efetividade da intervenção proposta para minimização dos efeitos do comprometimento cognitivo leve sobre variáveis cognitivas como a memória de trabalho, velocidade de processamento de informação e potência de ondas cerebrais.

Todavia, a interveniência promovida pelo isolamento social que limitou o período de intervenção à apenas 7 semanas pode ter sido determinante para a falta de significância estatística encontrada, pois todos os dados descritivos mostraram diferenças percentuais que permitem supor que os participantes do grupo experimental sofreram efeitos mais positivos nas variáveis avaliadas quando comparados aos participantes do grupo controle. Uma vez que, nas variáveis que indicam melhora da função cognitiva o desempenho do grupo experimental foi melhor, e, mesmo quando os resultados apontaram piora da função cognitiva, tal efeito foi menos intenso nos idosos do grupo experimental.

Sendo assim, levando-se em conta os dados produzidos pode-se presumir que a intervenção verificada no presente estudo mostrou resultados ao menos promissores para uma proposta de intervenção não farmacológica para o retardamento dos efeitos do comprometimento cognitivo leve, levantando a possibilidade para que metodologias semelhantes possam ser testadas com diferentes tipos de exercícios com alta demanda cognitiva associados à estimulação cerebral não invasiva por períodos superiores a 7 semanas de intervenção.

Persp. Online: biol. \& saúde, Campos dos Goytacazes, 37 (11) 36-49, 2021 seer.perspectivasonline.com.br 


\section{REFERENCIAS}

ARÊAS NETO, N.T.; et al. Estimulação cortical: efeitos agudos sobre variáveis bioperacionais em jogadores armadores de basquetebol. Revista Digital EFdeportes.com, v. 15, p. 150, 2010.

BRANCO, L. P. A. et al. Desempenho no futebol: estimulação cerebral e neurônios espelho. Biológicas \& Saúde, v. 10, n. 34, p. 16-32, 2020.

CALOMENI, M.R. et al. Brain stimulation used as biofeedback training for recovery of motor functions deteriorated by stroke. Arquivos de neuro-psiquiatria, v. 71, n. 3, p. 159-164, 2013.

CALOMENI, M. R. et al. Treinamento Mental e Estimulação Cerebral: Efeitos na Memória de Trabalho de Crianças com Dificuldade de Aprendizagem. Humanas \& Sociais Aplicadas, v. 8, n. $22,2018$.

CALOMENI, M. R. et al. Modulatory effect of association of brain stimulation by light and binaural beats in specific brain waves. Clinical practice and epidemiology in mental health: CP \& EMH, v. 13, p. 134, 2017.

CAMARANO, A. A.; KANSO, S.; FERNANDES, D. Envelhecimento populacional, perda de capacidade laborativa e políticas públicas. 2013.

CARANELli, P. et al. Diagnóstico de doença de Alzheimer no Brasil. Exames complementares. Dementia \& Neuropsychologia, v. 5, n. 1, p. 11-20, 2011.

CARIELO, A.A. et al. Acute effects of brain stimulation in short-term memory of young persons. Academia Arena, v. 2, n. 3, p. 5-14, 2010.

COELHO, F. G. M. et al . Atividade física sistematizada e desempenho cognitivo em idosos com demência de Alzheimer: uma revisão sistemática. Rev. Bras. Psiquiatr., São Paulo , v. 31 n. 2, p. 163-170, June 2009 . Available from <http://www.scielo.br/scielo.php?script=sci_arttext\&pid=S1516-

44462009000200014\&lng=en\&nrm=iso>. access on $16 \quad$ Nov. 2020. https://doi.org/10.1590/S1516-44462009000200014

CONSORTIUM, B. Randomized trial on the effects of a combined physical/cognitive training in aged MCI subjects: the Train the Brain study. Scientific Reports, v. 7, p. 39471, 2017.

COSTA, A. R. D.; SEQUEIRA, C. Efetividade de um Programa de Estimulação Cognitiva em Idosos com Défice Cognitivo Ligeiro. Revista Portuguesa de Enfermagem de Saúde Mental, Porto , n. 9, p. 14-20, jun. 2013 . $<$ http://www.scielo.mec.pt/scielo.php?script=sci_arttext\&pid=S1647-

$21602013000100003 \& \operatorname{lng}=$ pt\&nrm=iso $>$. acessos em 12 nov. 2020.

ESCOSTEGUY, C.C. Tópicos Metodológicos e Estatísticos em Ensaios Clínicos Controlados Randomizados. Arquivos Brasileiros de Cardiologia, v. 72, n. 02. Rio de Janeiro, 1999.

GONÇALVES, D. et al. Avaliação das funções cognitivas, qualidade de sono, tempo de reação e risco de quedas em idosos institucionalizados. Estudos Interdisciplinares sobre o envelhecimento, v. 19, n. 1, p.95-108, 2014.

Persp. Online: biol. \& saúde, Campos dos Goytacazes, 37 (11) 36-49, 2021

seer.perspectivasonline.com.br 
JERÔNIMO, G.M. Envelhecimento sadio, Comprometimento Cognitivo Leve e doença de Alzheimer: um estudo das estratégias comunicativas na narrativa oral. Revista Letras de Hoje, v.53, n.1. Porto Alegre, Jan/Mar. 2018. http://dx.doi.org/10.15448/1984-7726.2018.1.28894.

JURCAK, V.; TSUZUKI, D.; DAN, I. 10/20, 10/10, and 10/5 systems revisited: their validity as relative head-surface-based positioning systems. Neuroimage, v. 34, n. 4, p. 1600-1611, 2007.

LIMA, A. P. et al. Avaliação da eficácia de um programa de estimulação cortical para melhora da atenção de crianças com TDAH. Saúde (Santa Maria), v. 40, n. 1, p. 71-76, 2014.

LIMA NETA, A.G. et al. Eficácia do treinamento cognitivo associado ao exercício físico em indivíduos com doença de Alzheimer: uma metanálise. Research, Society and Development, v. 9, n. 12, p. e15791211022-e15791211022, 2020.

LUCKHAUS, C. et al. Quantitative EEG in progressing vs stable mild cognitive impairment (MCI): results of a 1-year follow-up study. International Journal of Geriatric Psychiatry: A journal of the psychiatry of late life and allied sciences, v. 23, n. 11, p. 1148-1155, 2008.

MAIA, D. V. F. et al. Exercício Físico na Pessoa com Demência: Revisão Sistemática de Literatura. Revista Portuguesa de Enfermagem e Reabilitação, Porto, v2, n1, p. 27, 2019. Disponível em: https://www.aper.pt/ficheiros/revista/RPERv2n1.pdf\#page=27

MARLATS, F. et al. Comparison of effects between SMR/delta-ratio and beta1/theta-ratio neurofeedback training for older adults with Mild Cognitive Impairment:a protocol for a randomized controlled trial. Trials, v. 20, n. 1, p. 88, 2019.

MARQUES, L.J. et al. Padrão de atividade cortical ótima para aprendizagem hábil-motriz e cognitiva. Fitness \& Performance Journal, v. 5, n. 3, p.177-186, 2006.

MCEWEN, S. C. et al. Simultaneous aerobic exercise and memory training program in older adults with subjective memory impairments. Journal of Alzheimer's Disease, v. 62, n. 2, p. 795-806, 2018.

MINAYO, M.C.S., et al. Longevity:bonus or onus? Revista Scielo, v.24, n.01. Rio de Janeiro, 2019. Disponível em <https://doi.org/10.1590/1413-81232018241.31212018> Acesso em 07 ago 2019.

NASCIMENTO E. WAIS-III: manual para administração e avaliação. São Paulo: Casa do Psicólogo; 2004.

NETTO, T. M. et al. Efeito de um programa de treinamento da memória de trabalho em adultos idosos. Psicologia: Reflexão e Crítica, v. 26, n. 1, p. 122-135, 2013.

OKAMURA, H. et al. Combined exercise and cognitive training system for dementia patients: a randomized controlled trial. Dementia and geriatric cognitive disorders, v. 45, n. 5-6, p. 318-325, 2018. 
OKANO, A. H. et al. Estimulação cerebral na promoção da saúde e melhoria do desempenho físico. Revista Brasileira de Educação Física e Esporte, v. 27, n. 2, p. 315-332, 2013.

OKAZAKI, V.H.A. Software Reaction Time Task. v.2.0 2011. Disponível em: www.okazaki.webs.com. Acesso em 30 de abr. 2019.

ORCIOLI-SILVA, D. et al. A program of physical activity improves gait impairment in people with Alzheimer's disease. Motriz: Revista de Educação Física, v. 24, n. 1, 2018.

PAULA, J.J. et al. Propriedades psicométricas de um protocolo neuropsicológico breve para uso em populações geriátricas. Revista de Psiquiatria Clínica, v. 37, n.6, p.251-255, 2010. Disponível em: http://www.scielo.br/scielo.php?script=sci_arttext\&pid=S010160832010000600002\&lng=en\&nrm=iso. Acesso em: 16 de abr. 2018

PINEDA, A. M. Uso de redes complexas no estudo e no diagnóstico da Doença de Alzheimer. Dissertação apresentada à Universidade Estadual Paulista "Júlio de Mesquita Filho" para obtenção do título de mestre em Biometria. 2019.

RADIĆ, B. et al. EEG Analysis and Spect Imaging in Alzheimer's Disease, Vascular Dementia and Mild Cognitive Impairment. Psychiatria Danubina, v. 31, n. 1, p. 111-115, 2019.

ROSSATO, L. C.; CONTREIRA, A. R.; CORAZZA, S. T. Análise do tempo de reação e do estado cognitivo em idosas praticantes de atividades físicas. Fisioter. Pesqui., São Paulo, v. 18 , n. 1, p. 54-59, Mar. 2011 . Available from <http://www.scielo.br/scielo.php?script=sci_arttext\&pid=S1809-

29502011000100010\&lng=en\&nrm=iso $>$ access on 12 Nov. 2020. http://dx.doi.org/10.1590/S1809-29502011000100010.

SANTOS, S.; TANI, G. O. Tempo de reaçäo e a aprendizagem de uma tarefa de" timing" antecipatório em idosos. Rev. paul. educ. fís, p. 51-62, 1995.

SILVA VERNON, F. et al. Estimulação cerebral não invasiva e efeito sinérgico do exercício físico sobre a fragilidade e equilíbrio de idosos. Revista Eletrônica Acervo Saúde, v. 12, n. 4, p. e2602-e2602, 2020.

SILVA VERNON, F. et al. Brain stimulation used as biofeedback in neuronal activation of the temporal lobe area in autistic children. Arquivos de neuro-psiquiatria, v. 74, n. 8, p. 632-637, 2016.

SILVA VERNON, F. et al. Stimulation by Light and Sound: therapeutics effects in humans. systematic review. Clinical practice and epidemiology in mental health, v.26, n.11, p. 150$154,2015$.

SILVA VERNON, F. et al. Physical Training Coupled with Non-Invasive Brain Stimulation Modulates Cortical Waves Decreasing the Likelihood of Falls in Adults Elderly with Fragility. International Journal of Advanced Engineering Research and Science, v. 6, n. 5, 2019.

SOUSA, M. C. et al. O envelhecimento da população: aspectos do Brasil e do mundo, sob o olhar da literatura. Brazilian Journal of Development, v. 6, n. 8, p. 61871-61877, 2020.

Persp. Online: biol. \& saúde, Campos dos Goytacazes, 37 (11) 36-49, 2021 seer.perspectivasonline.com.br 
SOUTAR, R., LONGO, R. Doing neurofeedback: an introduction. San Rafael: ISNR Research Foundation, 2011, 202p.

STYLIADIS, C. et al. Neuroplastic effects of combined computerized physical and cognitive training in elderly individuals at risk for dementia: an eLORETA controlled study on resting states. Neural plasticity, 2015.

TEIXEIRA, I.L.N. et al. Qualidade de vida do cuidador familiar de idoso com alzheimer: uma revisão de literatura. Brazilian Journal of Health Review, v. 4, n. 2, p. 5221-5237, 2021.

THOMAS, A. et al. The effects of aerobic activity on brain structure. Frontiers in psychology, v. 3, p. 86, 2012. 